\title{
A Proposed Fetal Risk Scoring System for Gestational Diabetes to assist in optimizing Timing of Delivery
}

\author{
Ismail Bhorat ( $\square$ ismail@iebhorat.co.za )
}

University of KwaZulu-Natal College of Health Sciences https://orcid.org/0000-0002-1870-8459

\section{Tarylee REDDY}

Medical Research Council of South Africa: South African Medical Research Council

\section{Research article}

Keywords: cardiac Doppler, myocardial performance index, E/A ratio, gestational diabetes, macrosomia, polyhydramnios

Posted Date: February 25th, 2021

DOl: https://doi.org/10.21203/rs.3.rs-271807/v1

License: (c) (i) This work is licensed under a Creative Commons Attribution 4.0 International License.

Read Full License 


\section{Abstract}

Background: Gestational diabetes is characterized by three main factors:

macrosomia, increased metabolic rate and large vascular cross sections. A critical and crucial finding in diabetic pregnancies is that significant acidaemia and hyperlacticemia can occur in fetuses in the absence of hypoxaemia. The increased metabolic rate results in significant increases in oxidative metabolism but this capacity is reduced in fetuses due to low pyruvate dehydrogenase activity increasing the risk for acidosis. This pathophysiology is not recognized by standard monitoring models which revolves around placental insufficiency which is in fact not the problem in a gestational diabetic pregnancy. A proposed risk scoring system has been developed based on our previous studies to risk categorise gestational diabetics in terms of fetal outcome.

Methods: The diabetic cases from four case-control studies were combined to form a total sample of 159 cases for validation of the risk scoring system. Univariate logistic regression was used to assess the effect of individual risk factors with proposed cutoffs on adverse pregnancy outcome. The diagnostic accuracy of the total summative score, was assessed by computing the area under the ROC curve.

Results: Four potential parameters were identified to risk- categorise fetuses in a gestational diabetic pregnancy ie the myocardial performance index (MPI), E/A ratio (marker of diastolic dysfunction), increasing fetal weight (macrosomia) and increased amniotic fluid index (AFI). The total score, obtained by summation of the composite scores for parameters ranged from 0 to 11 . The total score performed as an excellent predictor of adverse outcome, evidenced by the ROC area under the curve of 0.94. A cutpoint of 6 on the score confers a sensitivity of $84.2 \%$ and specificity of $90.2 \%$ for detection of adverse outcome.

Conclusion: To our knowledge this is the first Gestational Diabetic Scoring system proposed to predict an adverse outcome.

\section{Background}

Gestational diabetes starts with an abnormal glucose tolerance in the mother causing maternal hyperglycaemia which triggers a sequence of events resulting in fetal hyperglycaemia and subsequently fetal hyperinsulinaemia [1, 2]. It is the hyperinsulinaemia in the fetus that forms the basis of the pathophysiology. Gestational diabetes is characterized by three main factors: macrosomia, increased metabolic rate and large vascular cross sections. A critical and crucial finding in diabetic pregnancies is that significant acidaemia and hyperlacticemia can occur in fetuses in the absence of hypoxaemia [3, 4]. The so-called "unexplained" stillbirths of diabetic pregnancies especially in the 3rd trimester could most likely be due to fetal acidaemia as a consequence of an increased metabolic rate. The increased metabolic rate results in significant increases in oxidative metabolism but this capacity is reduced in fetuses due to low pyruvate dehydrogenase activity increasing the risk for acidosis. This pathophysiology is not recognized by standard monitoring models which revolves around placental insufficiency which is in fact not the problem in a gestational diabetic pregnancy. In fact the most widely used antenatal 
surveillance technique in monitoring diabetic pregnancies is the umbilical artery Doppler velocimetry [5] which is a marker for placental insufficiency. Standard fetal monitoring models presently used in diabetic pregnancies are thus inappropriate, inadequate and insufficient as they do not answer the question of fetal compromise in gestational diabetes (in the absence of microvascular complications), rendering them ineffective. Our research group has investigated cardiac Doppler in particular the myocardial performance index (MPI) and E/A ratios, in a series of prospective studies in poorly controlled (PCD), well controlled (WCD) and impaired gestational diabetics (GIGT) as well as biophysical parameters and their possible links to adverse outcomes $[6,7,8,9]$ after having first established gestational age adjusted trends and reference ranges of the MPI in normal pregnancies [10]. Four parameters were identified in these studies that appeared to impact the diabetic pregnancy in terms of fetal outcome. These four parameters were elevated MPI's and decreasing E/A ratios (both cardiac Doppler parameters), macrosomia and increasing amniotic fluid indices (AFI). Using this data a scoring system was proposed to see if this could be used to risk- categorise gestational diabetics for fetal outcome which may be used as a tool in guiding clinicians to establishing optimal timing of delivery.

\section{Methods}

The gestational diabetic cases from four case-control studies $[6,7,8,9]$ by our research group were combined to form a total sample of 159 cases for validation of the risk scoring system. The recruitment, categorization of degree of gestational diabetes, methodology in performing the myocardial performance index and management of patients were consistent and standard across the studies and could be combined to form a total sample for validation of the risk scoring system. All studies were prospective cross sectional studies of the MPI in fetuses of gestational diabetic pregnancies conducted at the tertiary referral Fetal Unit at Inkosi Albert Luthuli Central Hospital in Durban, South Africa. All patients were in the 3rd trimester of pregnancy.

The cases included poorly or suboptimally controlled gestational diabetics, well controlled gestational diabetics and gestationally impaired glucose tolerance patients. All patients in the study groups were categorized by a combination of an oral glucose tolerance test, blood glucose profiles and measurement of glycosylated haemoglobin levels ( $\mathrm{HbA} 1 \mathrm{C} \%)$ within 4 weeks of the echocardiographic assessment.

Poor or suboptimal control was defined by suboptimal blood glucose profiles and measurement of glycosylated haemoglobin level ( $\mathrm{HbA} 1 \mathrm{C} \%)$ within 4 weeks of the echocardiographic assessment revealing poor or suboptimal blood glucose control of the study patients with an average HbA1C of 64 $\mathrm{mmol} / \mathrm{mol}$ [ $8 \%$ ] or more (National Institute for Health and Care Excellence NICE guidelines for gestational diabetes [Clinical Guideline 63] state that women with diabetes should aim to achieve an $\mathrm{HbA1C}$ level of $43.3 \mathrm{mmol} / \mathrm{mol}[6.1 \%]$ or lower). Two studies were performed in this category of gestational diabetics; in the first study 29 consecutive poorly controlled gestational diabetic women on insulin in the 3rd trimester were recruited matched with 29 normal controls[6] and in the 2nd study 44 consecutive poorly controlled women on insulin were recruited in the 3rd trimester matched with 44 controls[7]. 
Well controlled gestational diabetics were defined firstly by the WHO 's criteria of a $2 \mathrm{hr}$ level $>7.8 \mathrm{mmol} / \mathrm{I}$ and $<11.0 \mathrm{mmol} / \mathrm{I}$ after $75 \mathrm{~g}$ oral glucose tolerance test (OGTT) in the 3rd trimester [11] and on review of self monitoring blood glucose levels achieving $2 \mathrm{hr}$ post-prandial levels of $<7 \mathrm{mmol} / \mathrm{l}$ and pre-prandial levels $<5.5 \mathrm{mmol} / \mathrm{l}$. All patients in the well-controlled gestational diabetic group had $\mathrm{HbA} 1 \mathrm{c}$ results of $<6 \%$ reflecting good control. The well- controlled group achieved good control on medication either metformin or insulin. 54 consecutive well controlled women were recruited in the 3rd trimester with matched controls.

GIGT patients were defined firstly by the WHO 's criteria of a $2 \mathrm{hr}$ level $>7.8 \mathrm{mmol} / \mathrm{l}$ and $<11.0 \mathrm{mmol} / \mathrm{I}$ after $75 \mathrm{~g}$ oral glucose tolerance test (OGTT) in the 3rd trimester[11] and on review of self monitoring blood glucose levels achieving $2 \mathrm{hr}$ post-prandial levels of $<7 \mathrm{mmol} / \mathrm{I}$ and pre-prandial levels $<5.5 \mathrm{mmol} / \mathrm{l}$. All patients in the GIGT group had HbA1c results of $<6 \%$ reflecting good control. In this group control was achieved only on diet. Thirty-two consecutive women defined as GIGT were recruited in the 3rd trimester with matched controls.

The mean gestational age at which MPI was performed in all studies was between 33-35 weeks gestation. All studies were prospective and cross-sectional.

All the different gestational diabetic categories had control groups that were randomly selected from the antenatal clinic and who were not diabetic as defined by the WHO criteria of a $2 \mathrm{hr}$ level $<7.8 \mathrm{mmol}$ after a $75 \mathrm{~g}$ OGTT[11]. The controls were matched for gestational age, maternal age, parity, gravidity, BMI and past obstetric history at inclusion in all the patients.

All pregnancies in all studies in both the study and control groups were spontaneously conceived.

Pregestational diabetics according to history were excluded from recruitment to exclude possible microvascular complications which could be a confounding variable and to give conformity to the study groups. Exclusion criteria in all studies were multiple pregnancies, congenital malformations, evidence of placental mediated disease and abnormal fetal heart rates (either tachycardia or bradycardia). Placental mediated disease was defined by either the presence of growth restriction $(A C<10$ th percentile for gestational age with an elevated umbilical artery resistance index $>90$ th percentile for gestational age) and/or presence of pre-eclampsia as defined by a blood pressure of greater than $140 / 90 \mathrm{mmHg}$ with proteinurea[12].

Ethical approval for all studies was obtained from the Biomedical Research Ethics Committee at the University of Kwa-Zulu Natal. All studies were performed in accordance with the Declaration of Helsinki. All participants were adult over 18 years. All participants provided written informed consent to participate in this study.

Fetal echocardiography using either an E8 General Electric Voluson Ultrasound system (GE Medical Systems WI, USA) or Siemens Antares ultrasound system (Siemens Medical Systems Malvern PA, USA) was performed in each woman in all studies. The four chamber view,outflow tract views, triple vessel 
view, longitudinal view of the aortic arch and colour flow mapping were used to screen for cardiac malformations.

The MPI in all studies was calculated in the fetal left ventricle[10, 13]. Our previous study established reference intervals and trends of the MPI in normal pregnancies and the methodology of obtaining the MPI has been described in detail in the paper[10]. A cross sectional image of the fetal thorax at the level of the 4-chamber view with an apical projection of the heart was obtained. The Doppler sample was opened to $3 \mathrm{~mm}$ and placed in the internal leaflet of the mitral valve (MV). In this location owing to its closeness to the aortic valve (AV), the opening and closing AV clicks were registered. The angle of insonation was always $<30$ degrees. E/A waveform was always displayed as positive flow. The Doppler gain was lowered as far as possible to clearly visualize the echoes corresponding to the opening and closing clicks of the two valves at the beginning and at the end of the mitral valve and aortic waveforms. The peak of the valve clicks was used in the measurement of the time intervals rather than the base, as it is a clearer landmark, overcoming variations in valve click width and resulting in better reproducibility [10, $14,15]$. The Doppler sweep velocity was set at $5 \mathrm{~cm} / \mathrm{sec}$ and wall motion filter at $300 \mathrm{~Hz}$. The three time periods were estimated as follows :Isovolumetric contraction time (ICT) from beginning of MV closure to AV opening; Ejection time (ET) from AV opening to closure;Isovolumetric relaxation time (IRT) from AV closure to MV opening.The Mod-MPI = (ICT + IRT) /ET. We have previously documented high levels of inter- and intraobserver variability agreement for the MPI and its components in our paper establishing reference intervals of mod-MPI in normal pregnancies [10].

In addition to the echocardiographic data, sonographic data including estimated fetal weights and amniotic fluid indices were determined and recorded. The umbilical artery (UA) resistance (RI) and pulsatility index $(\mathrm{PI})$, middle cerebral artery (MCA) pulsatility index $(\mathrm{PI})$ and ductus venosus pulsatility index $(\mathrm{PI})$ were also determined in both groups. Cerebroplacental ratio was then determined.

All patients in all studies were delivered according to existing standard protocols for diabetic pregnant patients at our institution, which includes non-reassuring fetal cardiotocogram (CTG) findings, poor biophysical profiles, or persistent elevations of UA RI/PI. If fetal monitoring was satisfactory, delivery was delayed until 39-40 weeks after which the patient would be induced or caesarean section performed for obstetrical reasons.

Pregnancy outcomes were recorded in both groups. An abnormal outcome in our studies was defined as any one of the following: stillbirth, neonatal death, tachypnoea with pulmonary oedema, neonatal hypoglycaemia, neonatal cord $\mathrm{pH}<7.2,5$ min Apgar score $<7$, polycythaemia and nucleated red blood cells $>10 / 100$ white blood cell counts (markers for hypoxia), cardiomyopathy, NICU admissions.

Cardiac Doppler data were not used by clinicians in the management of the diabetic patients.

For details of methodology of each study please refer to the individual published studies $[6,7,8,9]$.

\section{Statistical Analysis}


Univariate logistic regression was used to assess the effect of individual risk factors with proposed cutoffs on adverse pregnancy outcome. The diagnostic accuracy of the total summative score, was assessed by computing the area under the ROC curve and by examining the sensitivity and specificity at key cutpoints of interest.

\section{Results}

A total of 159 fetuses of gestational diabetic pregnancies were studied ranging from 31 to 38 weeks gestation of which 32 cases (20\%), 73 cases $(46 \%)$ and 54 cases $(34 \%)$ were classified as gestationally impaired glucose tolerance (GIGT), poorly controlled gestational diabetics (PCD) and well controlled gestational diabetics (WCD) respectively. For details of the results of each study please refer to the individual published studies. A summary of the distribution of key risk factors in the 159 fetuses is presented in Table 1. The aforementioned variables were further categorized based into three or four category ordinal variables. E/A ratios under 0.60 were observed in $8.8 \%$ of the cases. The majority of cases presented with E/A ratios greater than 0.65 . MPI values exceeding a cutoff of 0.67 were observed in $21(13.2 \%)$ of fetuses. $65 \%, 24 \%$ and $12 \%$ of fetuses had AFI measurements under $20 \mathrm{~cm}$, between $20 \mathrm{~cm}$ and $25 \mathrm{~cm}$ and exceeding $25 \mathrm{~cm}$ respectively.

Table 1

Distribution of key risk factors

\begin{tabular}{|lll|}
\hline Parameter & Mean & SD \\
\hline AFI $(\mathrm{cm})$ & 19.54 & 11.97 \\
\hline EWF $(\mathrm{g})$ & 2585.40 & 472.79 \\
\hline Mod-MPI z-score & 4.02 & 1.39 \\
\hline E/A & 0.67 & 0.07 \\
\hline $\begin{array}{l}\text { Legend: AFI, amniotic fluid index; EWF expected weight of fetus; MPI myocardial performance index; } \\
\text { EA, E/A ratio. }\end{array}$ & \\
\hline
\end{tabular}

The relationship between each of the categories of these variables and adverse outcome is presented in Table 2 and Figs. 1a to 1d. Fetuses with an expected weight of fetus (EWF) greater than the 95th percentile had a 14 times higher odds of adverse outcome compared to those with EWF in the 50th -75th percentile range. In all E/A ratio and MPI categories of higher severity, highly statistically significant increases in the odds of adverse outcome were observed. Fetuses with an AFI between $20 \mathrm{~cm}$ and $25 \mathrm{~cm}$ were more than twice as likely to experience adverse outcomes ( $p$-value 0.026). This risk was significantly higher for fetuses with $\mathrm{AFI}$ greater than $25 \mathrm{~cm}$ where the odds of adverse outcome were approximately three times higher than those with AFI lower than $20 \mathrm{~cm}$ ( $p$-value 0.013 ). 
Table 2

Relationship between each of the categories of the variables and adverse outcome

\section{Abnormal outcome Total}

\begin{tabular}{|c|c|c|c|c|c|}
\hline & $\mathrm{n}$ & $\%$ & $\mathrm{~N}$ & Odds ratio & $\mathrm{p}$-value \\
\hline \multicolumn{6}{|c|}{ EWF Category (g) } \\
\hline 50-75th & 4 & 12.9 & 31 & & \\
\hline$<50$ th & 8 & 22.9 & 35 & $2(0.54-7.44)$ & 0.301 \\
\hline 75-95th & 12 & 27.3 & 44 & $2.53(0.73-8.77)$ & 0.143 \\
\hline$>95$ th & 33 & 67.3 & 49 & $13.92(4.16-46.59)$ & $<0.001$ \\
\hline \multicolumn{6}{|c|}{ E/A Category } \\
\hline$>=0.70$ & 3 & 3.7 & 81 & & \\
\hline $0.65-0.69$ & 7 & 41.2 & 17 & $18.2(4.0-81.91)$ & $<0.001$ \\
\hline $0.60-0.64$ & 34 & 72.3 & 47 & 68 (18.19-254.15) & $<0.001$ \\
\hline$<0.60$ & 13 & 92.9 & 14 & $338(32.62-3502.18)$ & $<0.001$ \\
\hline \multicolumn{6}{|c|}{ MPI z-score category } \\
\hline$<4$ & 7 & $10.4 \%$ & 67 & & \\
\hline $4-4.5$ & 12 & $35.3 \%$ & 34 & $4.67(1.63-13.39)$ & 0.004 \\
\hline $4.5-5$ & 22 & $59.5 \%$ & 37 & $12.57(4.53-34.92)$ & $<0.001$ \\
\hline$>=5$ & 16 & $76.2 \%$ & 21 & $27.43(7.68-97.99)$ & $<0.001$ \\
\hline \multicolumn{6}{|c|}{ AFI Category (cm) } \\
\hline$<20$ & 29 & 27.9 & 104 & & \\
\hline $20-25$ & 17 & 47.2 & 36 & $2.31(1.06-5.06)$ & 0.036 \\
\hline$>=26$ & 11 & 57.9 & 19 & $3.56(1.30-9.73)$ & 0.013 \\
\hline
\end{tabular}

Legend: EWF, expected weight of fetus; EA, E/A ratio; MPI myocardial performance index, AFI amniotic fluid index

The total score, obtained by summation of the composite scores for MPI, E/A ratio, EWF and AFI, ranged from 0 to 11. The distribution of this score is presented by outcome in Fig. 2. It is clear that the majority of fetuses with normal outcomes had risk scores lower than 5.

The total score performed as an excellent predictor of adverse outcome, evidenced by the ROC area under the curve of 0.94 (Fig. 3 and Table 3). A cutpoint of 6 on the score confers a sensitivity of $84.2 \%$ and 
specificity of $90.2 \%$ for detection of adverse outcome.

Table 3: Detailed report of sensitivity and specificity of the scoring system for prediction of adverse outcome

Correctly

Cutpoint Sensitivity Specificity Classified LR+ LR-

$\begin{array}{llllll}(>=0) & 100.00 \% & 0.00 \% & 35.85 \% & 1.0000 & \\ (>=1) & 100.00 \% & 22.55 \% & 50.31 \% & 1.2911 & 0.0000 \\ (>=2) & 100.00 \% & 43.14 \% & 63.52 \% & 1.7586 & 0.0000 \\ (>=3) & 98.25 \% & 63.73 \% & 76.10 \% & 2.7084 & 0.0275 \\ (>=4) & 96.49 \% & 76.47 \% & 83.65 \% & 4.1009 & 0.0459 \\ (>=5) & 92.98 \% & 83.33 \% & 86.79 \% & 5.5789 & 0.0842 \\ (>=6) & 84.21 \% & 90.20 \% & 88.05 \% & 8.5895 & 0.1751 \\ (>=7) & 63.16 \% & 94.12 \% & 83.02 \% & 10.7368 & 0.3914 \\ (>=8) & 47.37 \% & 98.04 \% & 79.87 \% & 24.1579 & 0.5368 \\ (>=9) & 28.07 \% & 98.04 \% & 72.96 \% & 14.3158 & 0.7337 \\ (>=10) & 12.28 \% & 99.02 \% & 67.92 \% & 12.5263 & 0.8859 \\ (>=11) & 5.26 \% & 100.00 \% & 66.04 \% & & 0.9474 \\ (>11) & 0.00 \% & 100.00 \% & 64.15 \% & 1.0000\end{array}$

ROC -Asymptotic Normal--

Obs Area Std. Err. [95\% Conf. Interval] 
Figure 3 demonstrates the ROC curve of total score as a prediction of adverse outcome. The adverse outcome rate in fetuses with scores of 3 or lower, $4-5,6-7$ and 8 or greater was $2.5 \%$. 33.33\%, $72.41 \%$ and $93.1 \%$ respectively (Table 4 ).

Table 4

Adverse outcome rate for each total score category

\begin{tabular}{|llll|}
\hline Total score & Normal outcome & Adverse outcome & Total \\
\hline$<=3$ & $78(97.5 \%)$ & $2(2.5 \%)$ & 80 \\
$4-5$ & $14(66.67 \%)$ & $7(33.33 \%)$ & 21 \\
$6-7$ & $8(27.59 \%)$ & $21(72.41 \%)$ & 29 \\
$8+$ & $2(6.9 \%)$ & $27(93.1 \%)$ & 29 \\
\hline
\end{tabular}

The complete scoring system with risk-categorisation is outlined in Table 5.

Table 5: Proposed scoring system in Gestational Diabetes for adverse outcome prediction 
3 points 2 points $\quad 1$ point $\quad 0$ points

\begin{tabular}{lllll} 
MPI (Z-scores) & $>5$ & $4.5-5$ & $4-4.5$ & $<4$ \\
E/A ratio & $<0.6$ & $0.6-0.64$ & $0.65-0.69$ & $>0.7$ \\
EWF $(\mathrm{g})$ & $>\mathrm{p} 95$ & $\mathrm{p} 75-\mathrm{p} 95$ & $<\mathrm{p} 50$ & $\mathrm{p} 50-\mathrm{p} 75$ \\
$\mathrm{AFI}(\mathrm{cm})$ & & $>26$ & $20-25$ & $<20$ \\
\hline
\end{tabular}

\section{Gestational Diabetic Score}

$\begin{array}{lll}0-3 \text { points } & \text { low risk } & \text { (LR 1-2) } \\ 4-5 \text { points } & \text { mild risk } & \text { (LR 4-5) } \\ \text { 6-7 points } & \text { moderate risk } & (\text { LR 8-10) } \\ >=8 \text { points } & \text { severe risk } & (L R>10)\end{array}$

Legend:MPI myocardial performance index, E/A early diastolic filling and late diastolic filling, IVRT

$(\mathrm{cm} / \mathrm{sec})$ isovolumetric relaxation time, EWF (g) expected weight of fetus, AFI $(\mathrm{cm})$ amniotic fluid index. LR

likelihood ratio

\section{Discussion}

In a series of prospective studies $[6,7,8,9]$ we identified four parameters that could potentially be used in a scoring system to risk categorise gestational diabetics in terms of fetal outcome and serve as a guide to clinicians managing gestational diabetics to optimize timing of delivery. Our score is based on the myocardial performance index (MPI), E/A ratio (marker of diastolic dysfunction), increasing fetal weight (macrosomia) and increased amniotic fluid index (AFI). Based on these four parameters a gestational diabetic score is proposed in predicting an adverse fetal outcome, risk- categorising the fetal condition into four groups from low risk to high risk. These four parameters probably reflect different pathophysiological mechanisms inherent in a fetus in a gestational diabetic pregnancy (without microvascular complications) and as a combination should give a more or less holistic view of the fetal condition. The total score, obtained by summation of the composite scores for MPI, E/A ratio, EWF and $\mathrm{AFI}$, range from 0 to 11. The total score performed as an excellent predictor of adverse outcome, as evidenced by the ROC area under the curve of 0.94 . A cutpoint of 6 on the score confers a sensitivity of $84.2 \%$ and specificity of $90.2 \%$ for detection of adverse outcome. 
Our first study in this area of investigation showed that the MPI was significantly increased and the E/A ratios significantly lower in fetuses of poorly controlled diabetic mothers compared with controls [6]. A total of 17 out of 25 fetuses with an elevated MPI showed abnormal outcomes. Of significance in this study was that adverse outcomes appeared to be related to the severity of an abnormal MPI. All control births had a normal outcome. This was corroborated in a follow up study investigating 44 poorly controlled gestational diabetics[7]. Adverse outcomes in this study were observed in 20 out of the 44 cases in the diabetic group, which corresponded to an adverse outcome rate of $45 \%$ with a similar correlation between increasing MPI values and adverse outcome. All control births had a normal outcome. We also showed that even in milder forms of gestational diabetes there is a percentage of patients whose fetuses are sensitive to the impaired glucose tolerance and these pregnancies can behave adversely despite the clinical label of mild disease resulting in a higher than an expected rate of adverse outcome in the gestational impaired glucose tolerance (GIGT) group of 25\% [8] as well as in the wellcontrolled gestational diabetic group with an adverse outcome rate of $22 \%[9]$. Macrosomia and polyhydramnios were also noted to be associated with a significantly higher risk for adverse outcome in these studies $[6,7,8,9]$.

Judging from the link between abnormal cardiac function and adverse outcomes in gestational diabetic pregnancies as well as demonstrating "hypoxic" markers viz polycythaemia and increased nRBC' (reflecting evidence of tissue hypoxia) in worsening cardiac function[7], it is reasonable to hypothesise that fetal cardiac dysfunction appears to predict abnormal metabolic mileus (and possible metabolic shifts) in diabetic pregnancies and that direct myocardial depression from the "hypoxia" leads to global cardiac dysfunction as reflected in the increased MPI. The concept of a "hypoxic" state in gestational diabetes is based on the fact that hyperinsulinaemia results in an increased metabolic rate which results in increased glucose oxidation and oxygen consumption but the capacity for oxidative metabolism is reduced in fetuses due to low pyruvate dehydrogenase activity and the risk for anaerobic metabolism increases irrespective of the prevailing po2 in the fetal circulation, thus increasing the risk for fetal acidosis. Macrosomia reflects the degree of fetal hyperinsulinaemia and the polyhydramnios reflects fetal osmotic diuresis and is indicative of suboptimal control. A Gestational Diabetic Score of $0-3$ points conferred a low risk status (likelihood ratio of 1-2 for an adverse outcome); 4-5 points, mild risk (likelihood ratio of 4-5 for an adverse outcome); 6-7 points, moderate risk (likelihood ratio of 8-10 for an adverse outcome ); >=8 points, severe risk (likelihood ratio of $>10$ for an adverse outcome). The majority of fetuses with normal outcomes had risk scores lower than 5 . The majority of fetuses with scores $>8$ had adverse outcomes. A suggested clinical way of using the scoring system based on our clinical experience, notwithstanding that each case has its own set of clinical variables which needs to be accounted for, would be the following:

Low risk - expectant management

Mild risk - delivery at 38 weeks with close feto-maternal monitoring from 36 weeks with cardiotocography and biophysical profiles once or twice weekly and fetal kick count charts. Patient can be managed as an outpatient also focussing on optimal blood glucose control. 
Moderate risk: delivery not later than 37 weeks with close fetomaternal monitoring from 35 weeks with cardiotocography, biophysical profiles twice weekly and fetal kick count charts and optimal blood glucose control. Patient can be managed as an outpatient if medical facilities and care is easily accessible.

Severe risk: these patients should be monitored as an inpatient from 34 weeks with cardiotocography daily, biophysical profiles every 3rd day, optimal blood glucose control and delivery not later than 36 weeks. Longitudinal MPI monitoring can be performed if there is access to echocardiographic facilities to better time delivery.

An algorithm describing the suggested clinical use of the scoring system is presented in Fig. 4.

This scoring system could also allow contingent scoring. In other words where fetal cardiac Doppler assessment expertise is not readily available contingent scoring could be undertaken, for example initially scoring on fetal weight and AFI (which are standard measurements), and if the patient scores high on this biophysical assessment, the patient could then be referred to a fetal unit for detailed cardiac Doppler assessment to complete the full scoring system. This scoring system could evolve with time and could be adapted depending on circumstances.

There are a number of reasons for the association of an elevated MPI and lower E/A ratios with adverse outcome. Impaired cardiac function and ventricular compliance especially diastolic dysfunction in fetuses of diabetic pregnancies is well documented $[16,17,18]$. Even in well controlled uncomplicated diabetic pregnancies ventricular septal thickness of up to $5 \mathrm{~mm}$ was observed at 35 weeks which provides some evidence that the level of metabolic control commonly achieved during pregnancy does not prevent progressive fetal myocardial thickening in a number of affected cases. This is certainly true for so-called well controlled or milder forms of gestational diabetes. Independent of the degree of glycaemic control, it has been demonstrated that hypertrophic cardiomyopathy with impaired cardiac function can complicate maternal diabetes $[20,21]$ which reiterates the point. There may already be the development of septal thickening before 20 weeks gestation. An increased preload index has been shown in the inferior vena cava of these fetuses which may be associated with a higher haematocrit at birth, increased neonatal morbidity and lower umbilical arterial blood $\mathrm{pH}$ [22]. In a recent study investigating stillbirths in diabetic pregnancies it has been reported that women with diabetes have a 14 times the risk of fetal cardiomyopathy identified at fetal autopsy as compared to women without diabetes [23]. This fact reemphasises the importance of cardiac function determination in fetuses of gestational diabetic pregnancies as a proxy for adverse outcome. Thus one of the main mechanisms inducing fetal compromise in gestational diabetes could be the development of myocardial dysfunction.

A diastolic dysfunction score was proposed by Zielinsky et al [24] in diabetic pregnancies using the septum primum excursion index, left atrial fractional shortening, mitral E/A ratio, pulmonary vein pulsatility index, ductus venosus pulsatility index, foramen ovale pulsatility index, aortic isthmus flow index and myocardial hypertrophy. This score still needs to be validated but its practicability in a clinical setting is questionable given the extent of the parameters that needs to be performed. Huhta et al [25] 
also proposed a cardiovascular score of 10 using venous Doppler, heart size, 4 valve filling assessing regurgitation and patterns of filling, fractional shortening on the ventricle and umbilical artery Doppler using positive flow, absent flow and reversed flow as parameters in the evaluation of heart failure in the fetus with and without hydrops. The Huhta score is useful in sick fetuses, probably not useful in gestational diabetes where the main pathophysiology is metabolic anomalies rather than placental insufficiency and so the umbilical artery Doppler component would not be useful, and probably not useful as a warning or predictive parameter of severity as the score seems more useful in an already established severe clinical state like hydrops.

In this study we have presented a statistically validated scoring system that risk-categorises a fetus in a pregnancy complicated by gestational diabetes. Further work, which requires a larger number of cases, includes a regression co-efficient weighted scoring system derived from multivariate analysis.

Strengths of the scoring system is that it is based on all prospective studies and due to consistency in recruitment, definition, methodology in performing fetal cardiac function and management it was possible to combine the studies in a validation study of a proposed risk scoring system. Limitations of the scoring system is that cardiac Doppler ie the MPI and E/A ratio require experience and training to obtain a reliable result. However this parameter shows very good reproducibility when its evaluation is performed using specific settings with valve clicks as landmarks as we have demonstrated in our study establishing reference intervals of the MPI in normal pregnancy [10]. A challenge would be to be able to transfer this assessment to more general units and to be sure that reliable results are being obtained. With commitment, practice and focus the MPI can be learned. The E/A ratio is an easier test to perform and also performs well independently in predicting an adverse outcome. Fetal weight estimation and amniotic fluid index determinations are standard measurements in ultrasound assessments.

\section{Conclusions}

To our knowledge this is the first Gestational Diabetic Scoring system, using cardiac Doppler and biophysical parameters, proposed to predict an adverse outcome, statistically validated and could potentially serve as a guide for clinicians to optimize timing of delivery in gestational diabetes as indicated above. Importantly the scoring system contributes to a new more scientific way of assessing a fetus in a gestational diabetic pregnancy, taking into account the pathophysiology inherent in these fetuses and moving away from standard monitoring models which are inappropriate and ineffective as they revolve mainly around placental insufficiency rather than metabolic anomalies which is the core pathophysiological problem in gestational diabetes.

\section{Declarations}

Authors' contributions: Prof Ismail Bhorat: Design, planning, conduct, data analysis and manuscript writingDr Tarylee Reddy: Design and data analysisAll authors approved the submitted version and agreed to be personally accountable for their contributions to the work. 
Funding: None to declare

Competing Interests: The authors declare they have no competing interests

Ethical Approval and consent to participate: All procedures performed in the studies were in accordance with ethical standards of the institutional research committee (Biomedical Research Ethics Committee at the University of Kwa-Zulu Natal) and with the 1964 Helsinki declaration and its later amendments. Informed consent was obtained from all individual participants included in the study.

Consent for publication: The authors affirm that human research participants provided informed consent for publication of any data.

Availability of data and material: Not applicable

Acknowledgements: None

Author details: ${ }^{1}$ Department of Obstetrics and Gynaecology, Subdepartment of Fetal Medicine, University of Kwa-Zulu Natal, Durban, South Africa; ${ }^{2}$ Biostatistics Unit, South African Medical Research Council of South Africa, Durban, South Africa

\section{References}

1. Salvesen DR, Brudenell JM, Proudler A, Crook D, Nicolaides KH. Fetal pancreaticbeta cell function in pregnancies complicated by maternal diabetemellitus. Am J Obstet Gynecol. 1993;168:1363-9.

2. Pedersen J. The Pregnant diabetic and her newborn. 2nd ed. Baltimore: Williams and Wilkins; 1977. pp. 211-20.

3. Bradley RJ, Brudenell JM, Nicolaides KH. Fetal acidosis and hyperlacticaemia diagnosed by cordocentesis in pregnancies complicated by maternal diabetes mellitus. Diabet Med. 1991;8:464-8.

4. Salvesen DR, Brudenell JM, Nicolaides KH. Fetal polycythaemia and thrombocytopenia in pregnancies complicated by maternal diabetes mellitus. Am J Obstet Gynecol. 1992;166:1287-92.

5. Wong SF, Chan FY, Cincotta RB. Use of umbilical artery Doppler velocimetry in the monitoring of pregnancy in women with pre-existing diabetes. Aust NZ JOnstet Gynecol. 2003;43:302-6.

6. Bhorat IE, Bagratee J, Pillay M, Reddy T. Use of the myocardial performance index as a prognostic indicator of adverse fetal outcome in poorly controlled gestational diabetic pregnancies. Prenat Diagn. 2014;34(13):1301-6.

7. Bhorat I, Foolchand S, Reddy T. Cardiac Doppler in poorly controlled gestational diabetics and its link to markers of hypoxia and adverse outcome. J Obstet Gynaecol. 2019. DOI.org/10.1080/01443615.2019.1710480.

8. Bhorat I, Pillay M, Reddy T. Determination of the fetal myocardial performance index in women with gestational impaired glucose tolerance and to assess whether this parameter is a possible 
prognostic indicator of adverse fetal outcome. Journal of Maternal-Fetal Neonatal Medicine. 2018;31(15):2019-26.

9. Bhorat I, Pillay M, Reddy T. Assessment of the fetal myocardial performance index (MPI) in well controlled gestational diabetics and to determine whether it is predictive of adverse perinatal outcomes. Paediatric Cardiology. 2019. DOI:10.1007/s00246-019-02158-4. PEDC-D-19-00153R1.

10. Bhorat IE, Bagratee J, Reddy T. Gestational age-adjusted trends and reference intervals of the myocardial performance index (Mod-MPI) with its interpretation in the context of established cardiac physiological principles. Prenat Diagn. 2014;34(11):1031-6.

11. Alberti K, Zimmett P. Definition, diagnosis and classification of diabetes mellitus and its complications. Part 1 Diagnosis and classification of diabetes mellitus provisional report of a WHO consultation. Diabet Med. 1998;15:539-53.

12. Figueras F, Gratacos E. Update on the diagnosis and classification of Fetal Growth Restriction and Proposal of a stage based Management protocol. Fetal Diagn Ther. 2014;36:86-98.

13. Hernandez-Andrade E, Figuero-Diesel H, Kottman C, et al. Gestational-age adjusted reference values for the modified myocardial performance index for evaluation of left fetal cardiac function. Ultrasound Obstet Gynecol. 2007;29:321-5.

14. Cruz-Martinez R, Figueras F, Bennasar, et al. Normal reference ranges from 11-14 week gestation of Fetal Left Modified Myocardial Performance Index by Concentional Doppler with the use of stringent criteria for delimitation of time periods. Fetal Diagn Ther. 2012;32:79-86.

15. Meriki N, Izurieta A, Welsh AW. Fetal left modified myocardial performance index: technical refinements in obtaining pulse Doppler waveforms. Ultrasound Obstet Gynecol. 2012;39:421-9.

16. Rizzo G, Aarduini D, Romanini C. Cardiac function in fetuses of type 1 diabetic mothers. Am J of Obstet Gynecol. 1991;164:837-43.

17. Rizzo G, Pietropolli A, Capponi A, et al. Analysis of factors influencing ventricular filling patterns in fetuses of type 1 diabetic mothers. J Perinat Med. 1994;22:149-57.

18. Weiner Z, Zloczower M, Lerner A, et al. Cardiac compliance in fetuses of diabetic women. Obstet Gynecol. 1999;93:948-51.

19. Jaeggi ET, Fouron JC, Proulx F. Fetal cardiac performance in uncomplicated and well controlled maternal type 1 diabetes. Ultrasound Obstet Gynecol. 2001;17:311-5.

20. Weber HS, Copel JA, Reece EA, Green J, Kleinman CS. Cardiac growth in foetuses of diabetic mothers with good metabolic control. J Pediatr. 1991;118:103-7.

21. Gandhi JA, Zhang XY, Maidman JE. Fetal cardiac hypertrophy and cardiac function in diabetic pregnancies. Am J Obstet Gynecol. 1995;173:1132-6.

22. Nicolaides KH, Rizzo G, Hecher K. Placental, Doppler F. London: The Parthenon Publishing Group, 2000: $128-129$.

23. Lynch TA, Westen E, Li D, et al. Stillbirth in women with diabetes: a retrospective analysis of fetal autopsy reports. Journal of Maternal- Fetal Neonatal Medicine. 2020. 
doi.org/10.1080.14767058.202017719213.

24. Zielinsky P, Luiz Piiccoli A. Myocardial hypertrophy and dysfunction in maternal diabetes. Early Hum Dev 20212; 88:273-8.

25. Huhta JC. Guidelines for the Evaluation of Heart Failure in the Fetus with or without hydrops. Pediatr Cardiol. 2004;25:274-86.

\section{Figures}
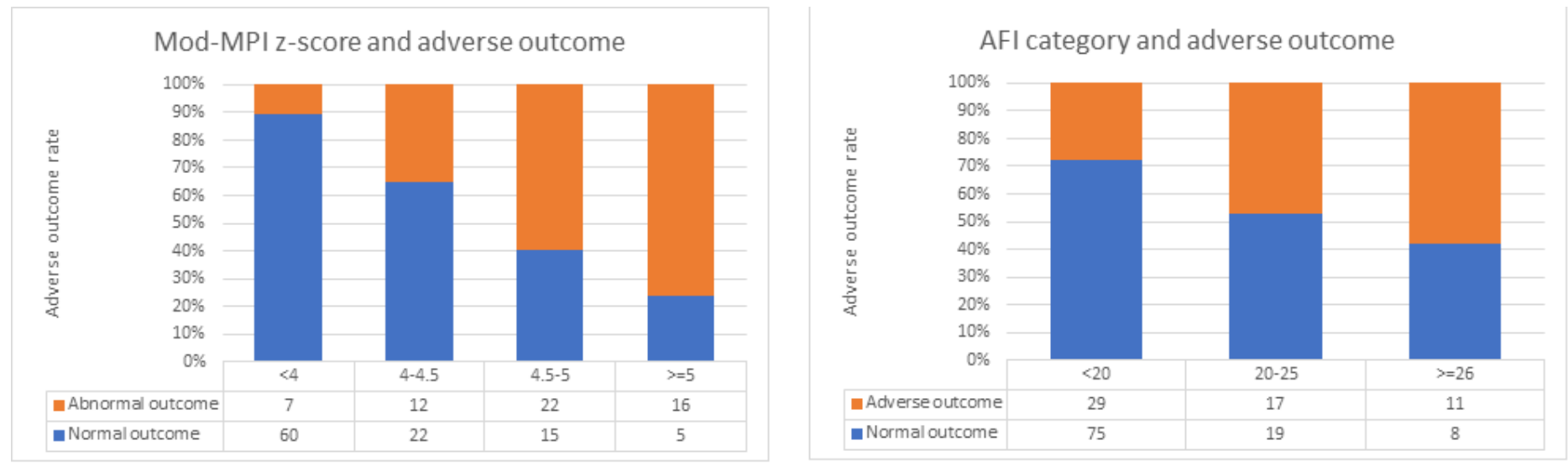

\section{A}

B
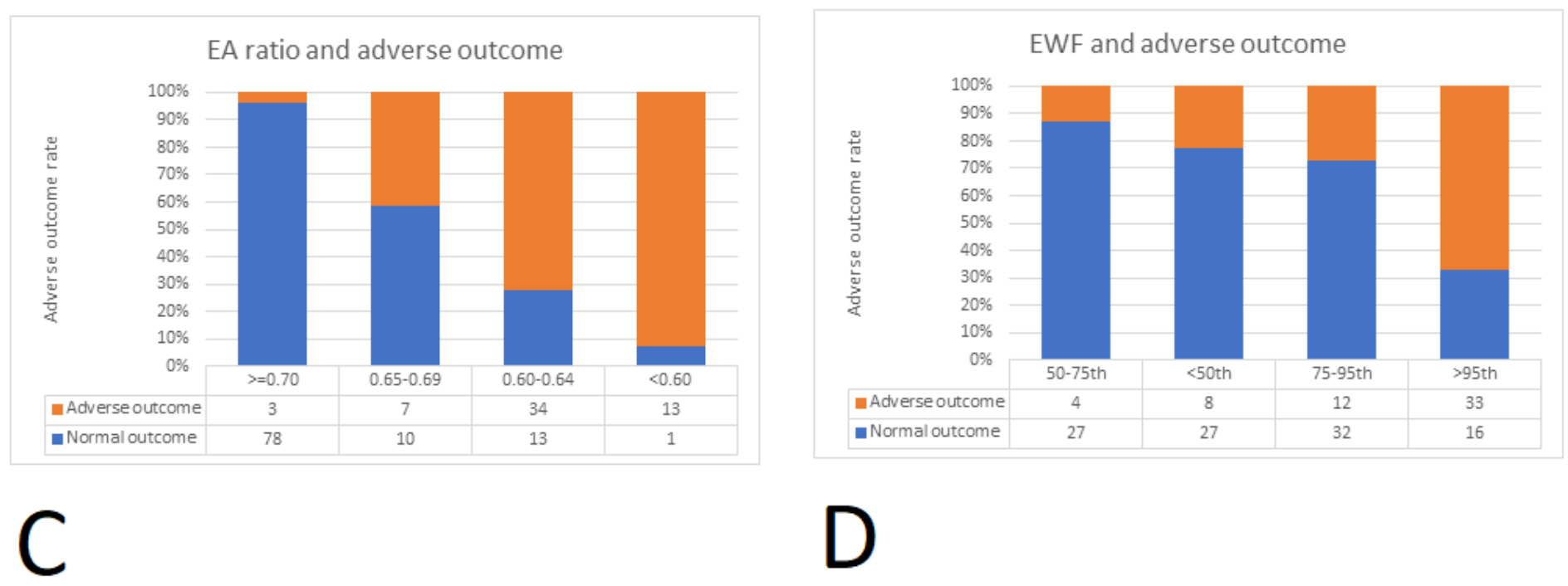

D

\section{Figure 1}

a: Myocardial performance index (MPI) and adverse outcome b: Amniotic fluid index (AFI) and adverse outcome c: E/A ratio and adverse outcome d: Expected weight of fetus (EWF) and adverse outcome 


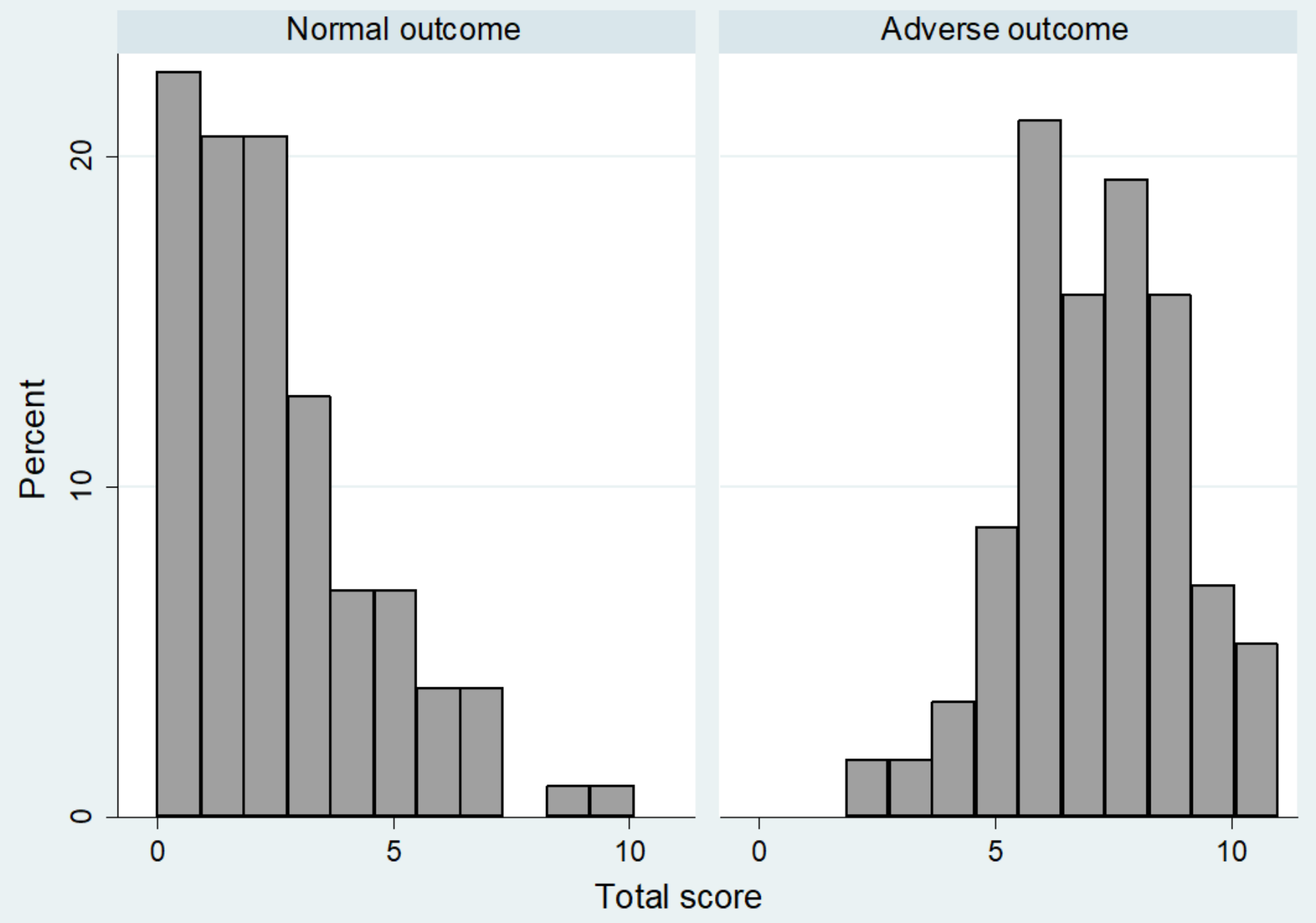

Figure 2

Distribution of total score by outcome 


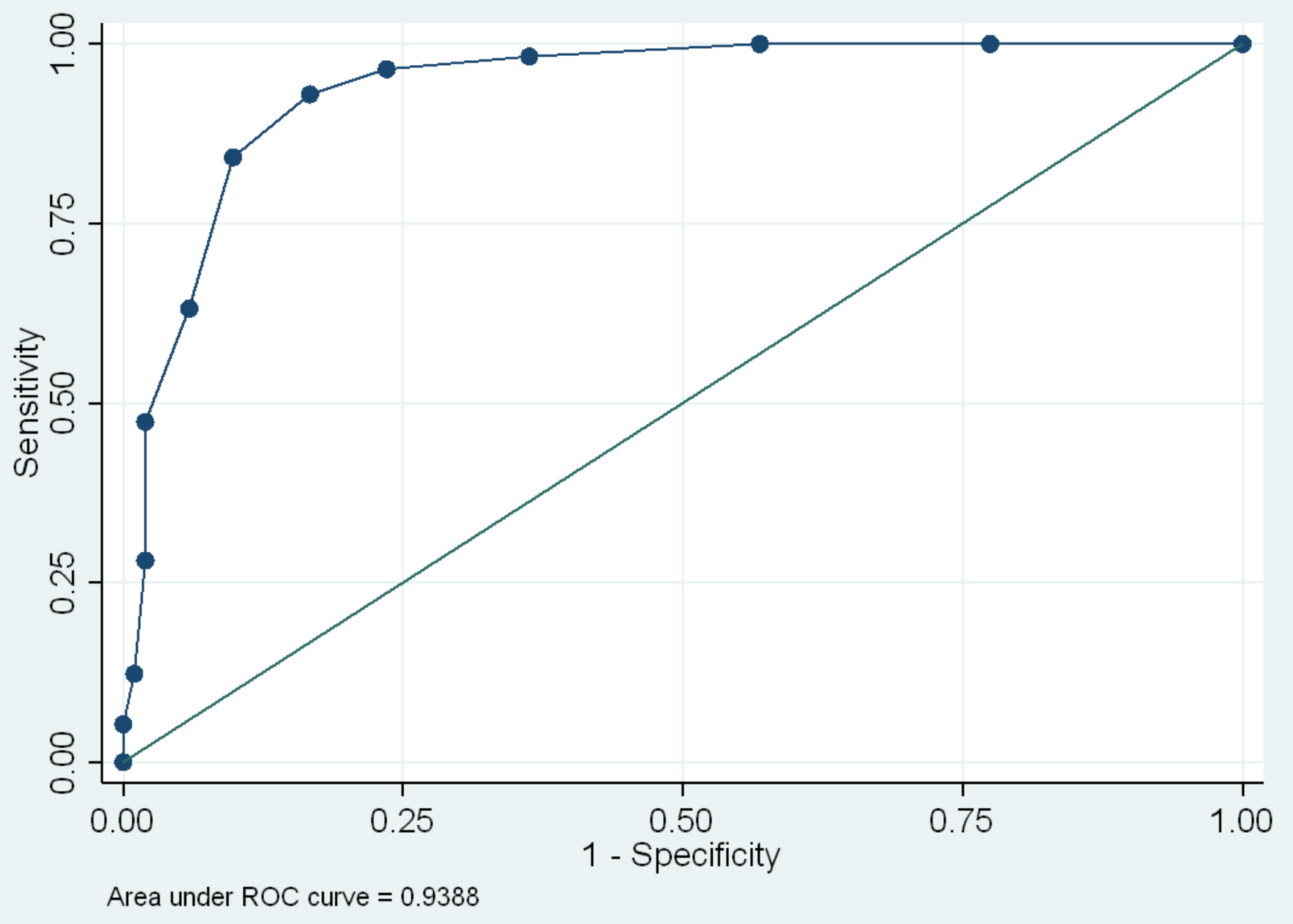

Figure 3

ROC curve of total score as a prediction of adverse outcome 


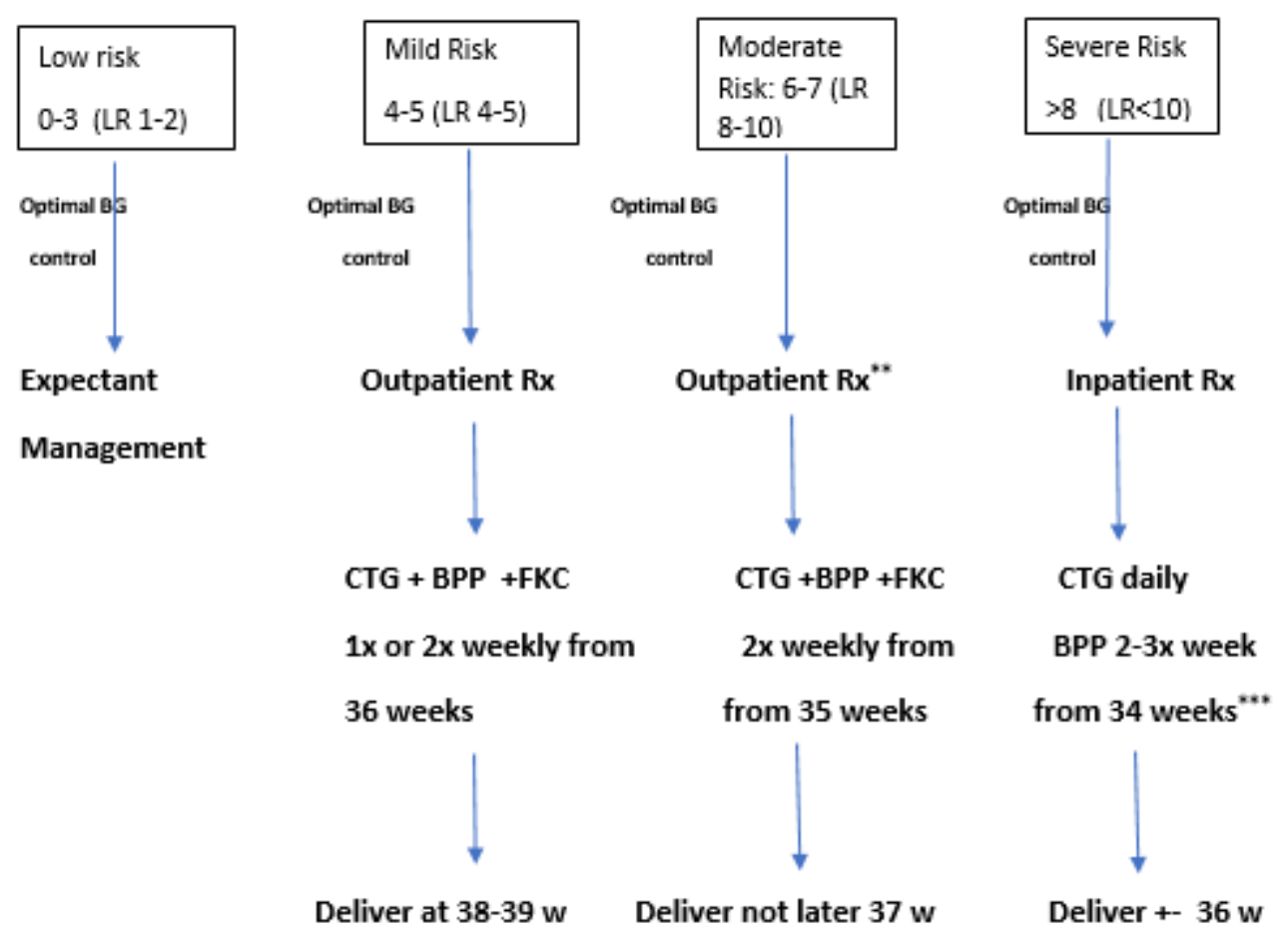

* Management needs to be individualised depending on the clinical variables

**Treat as outpatient depending on easy access to medical facilities

*** Longitudinal MPI monitoring if echocardiographic facility easily available

\section{Figure 4}

Clinical Algorithm using Proposed Gestational Diabetic Scoring System: 